\title{
Sinus histiocytosis with massive lymphadenopathy (Rosai-Dorfman disease) and cirrhosis of the liver: A case report and literature review
}

\author{
M K Kgomo, A A Elnagar, C Jeske, J Nagel \\ Dr Mpho Kgomo is a gastroenterologist and heads the Gastroenterology Division of Internal Medicine at Steve Biko Academic Hospital and the \\ School of Medicine, Faculty of Health Sciences, University of Pretoria, South Africa. He has an interest in oesophageal cancer research. Working \\ in the same division is Dr Ali Elnagar, a gastroenterologist with an interest in viral hepatitis. Dr Christian Jeske is a gastroenterologist in the \\ Gastroenterology Division of Surgery at Steve Biko Academic Hospital and the School of Medicine, Faculty of Health Sciences, University of Pretoria. \\ He has an interest in hepatobiliary diseases. Dr Jackoo Nagel is a specialist physician and a trainee gastroenterologist in the Gastroenterology \\ Division of Internal Medicine.
}

Corresponding author: M K Kgomo (kgomomk@worldonline.co.za)

Patients with cirrhosis of the liver usually present with a small, shrunken liver and a large spleen. The presence of an unusually huge liver should prompt the treating doctor to look for another cause, as this may be treatable and improve the patient's outcome. In South Africa tuberculosis and lymphoma in the presence of HIV infection should be excluded. Sinus histiocytosis with massive lymphadenopathy and cirrhosis is a rare combination and has not been reported before in the literature. This case is intended to make clinicians aware of this rare combination of diseases.

S Afr Med J 2016;106(5):463-464. DOI:10.7196/SAMJ.2016.v106i5.9614

\section{Case report}

A 62-year-old woman was known to have cirrhosis of the liver of unknown causation, confirmed histologically, with a micronodular pattern and regeneration. On previous investigation she was found to have ascites, and no varices on upper endoscopy. An autoimmune screen was positive for antinuclear factor (ANF) with a titre of 160 and a homogeneous pattern, but all other markers for autoimmune hepatitis were negative. Hepatitis B and C screening were also negative, iron status was normal, and copper and ceruloplasmin levels were normal.

The patient now presented to the liver clinic at Steve Biko Academic Hospital, Pretoria, South Africa, with massive hepatosplenomegaly. A computed tomography (CT) scan of the abdomen showed extensive adenopathy at the porta hepatis area (Fig. 1). Liver function tests showed an infiltrative pattern. The erythrocyte sedimentation rate (ESR) was elevated at $89 \mathrm{~mm} / \mathrm{h}$. Differential diagnoses of tuberculosis and lymphoma were considered.

A chest radiograph was normal, and laparoscopic lymph node biopsy was negative for both tuberculosis and lymphoma. However, it revealed prominent sinus histiocytosis, with the lymph node showing retained architecture and polarity.

The patient was continued on diuretics for cirrhosis. A search for other autoimmune diseases such as rheumatoid arthritis was negative. She will be continued on this treatment until the condition poses the risk of spleen rupture or threatens her life.

\section{Discussion}

As far as we could establish from a PubMed search, no other case of sinus histiocytosis and liver cirrhosis has been reported. The closest case is one of chronic hepatitis B viral infection and lymphoma with sinus histiocytosis reported by Melikyan et al. ${ }^{[1]}$ Sasaki et al. ${ }^{[2]}$ reported a case of sinus histiocytosis with massive lymphadenopathy complicated by pathological fractures, kidney failure and liver

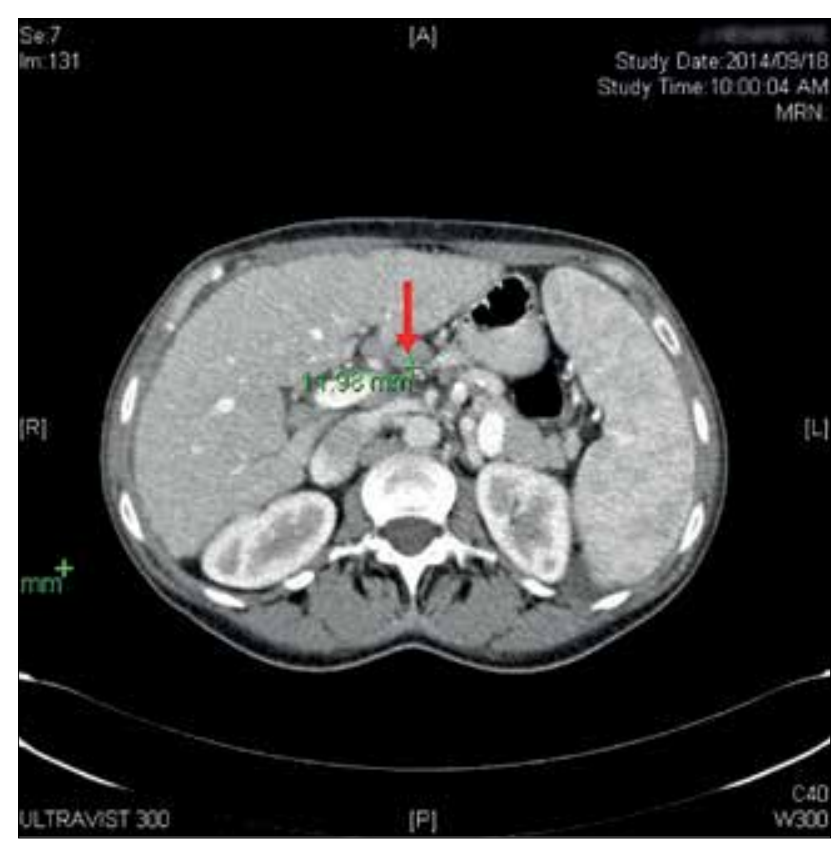

Fig. 1. CT scan of the abdomen of a patient with sinus histiocytosis and massive lymphadenopathy and cirrhosis of the liver showing lymph nodes in the porta hepatis area (arrow).

cirrhosis, treated with cladribine. In our case screening for both hepatitis $\mathrm{B}$ and $\mathrm{C}$ was negative, and apart from a positive test for ANF, no other explanation for the cirrhosis could be found.

Sinus histiocytosis with massive lymphadenopathy (SHML) is a rare and benign source of lymphadenopathy of unknown aetiology, first described in 1969 by Rosai and Dorfman (cited by Moore et $\left.a l .{ }^{[3]}\right)$, that can mimic neoplastic processes. ${ }^{[3]}$ It is a self-limiting 
disease that characteristically manifests as painless bilateral cervical adenopathy accompanied by fever, leucocytosis, an elevated ESR and hypergammaglobulinaemia. ${ }^{[4]}$ The most common sites for extranodal disease are the skin, ${ }^{[5]}$ upper respiratory tract and bone, followed by the genitourinary and lower respiratory tracts, oral cavity and soft tissues. ${ }^{[6]}$

The disease commonly presents in children and young adults, with supradiaphragmatic lymphadenopathy or extranodal lesions consisting of tissue infiltrates composed of a polyclonal population of sinus histiocytes. ${ }^{[3]}$

The disease involves overproduction of a type of white blood cell called non-Langerhans sinus histiocytes. These cells occur in lymph nodes, but can exist in other areas of the body and can lead to organ damage. The reason for the overproduction of these cells is not known, but several possibilities have been considered, including viral, bacterial, infective, environmental and genetic causes.

Treatment of SHML is unnecessary unless the disorder becomes life or organ threatening, since the disease will resolve spontaneously in most cases. ${ }^{[3]}$

SHML has been found to occur concomitantly with other diseases such as diffuse large B-cell lymphoma ${ }^{[1]}$ HIV infection, ${ }^{[2]}$ varicella zoster infection and autoimmune lymphoproliferative syndrome. ${ }^{[7]}$
Delacretaz et al. ${ }^{[8]}$ reported the first case of sinus histiocytosis and HIV, in a 31-year-old black woman with HIV infection who presented with symptomatic generalised lymphadenopathy. Since then a few other such cases have been reported, including that by Perry et al..$^{[9]}$ in 1998.

Other agents to treat the condition are still being researched, and case reports continue to appear, though at a slow rate. Because this disease is so rare, there have been no large-scale studies.

1. Melikyan AC, Kovringina AM, Gilyazildinova EA, Gittis MK. A case of sinus histiocytosis with massive lymphadenopathy in a patient with diffuse large cell lymphoma and chronic hepatitis B virus infection. Ter Arkh 2012;84(11):66-70.

2. Sasaki K, Pemmaraju N, Westin JR, et al. A single case of Rosai-Dorfman disease marked by pathological fractures, kidney failure, and liver cirrhosis treated by a single agent cladribine. Front Oncol 2014;29(4):297. DOI:103389/fonc.2014.00297

3. Moore JC, Zhao X, Nelson EL. Concomitant sinus histiocytosis with massive lymphadenopathy and diffuse large cell lymphoma: A case report. J Med Case Rep 2008;2:70. DOI:10.1186/1752-1947-2-70 diffuse large cell lymphoma: A case report. Med Case Rep 2008;2:70. DOI:10.1186/1752-1947-2-70
4. Park YK, Kim YW, Choi WS, Lim YJ. Sinus histiocytosis with massive lymphadenopathy, multiple skull involvement. J Korean Med Sci 1998;13(4):423-427.

5. Faucar E, Rosai J, Dorfman RF. Sinus histiocytosis with massive lymphadenopathy: Current status and future directions. Arch Dermatol 1988;124(6):1211-1214

6. Faucar E, Rosai J, Dorfman RF. Sinus histiocytosis with massive lymphadenopathy: Review of the entity. Semin Diagn Pathol 1990;7(1):19-73.

7. Silvers AR, Som PM, Meyer RJ. Egg shell nodal calcification in a patient with sinus histiocytosis with massive lymphadenopathy treated with interferon. Am J Neuroradiol 1996;17(2):361-363.

8. Delacretaz F, Meuge-Moraw C, Anwar D. Sinus histiocytosis with massive lymphadenopathy in a human immune deficiency virus-positive patient. Virchows Arch A 1991;419(3):251-254.

9. Perry BP, Gregg CM, Myers S, et al. Rosai-Dorman disease (extranodal sinus histiocytosis) in a patient with HIV. Ear Nose Throat J 1998;77(10):855-858.

Accepted 26 March 2016 